\title{
Trapping dipolar exciton fluids in GaN/(AIGa)N nanostructures
}

\author{
F. Chiaruttini, ${ }^{\dagger}$ T. Guillet, ${ }^{\dagger}$ C. Brimont, ${ }^{\dagger}$ B. Jouault,${ }^{\dagger}$ P. Lefebvre ${ }^{\dagger}$ J. Vives,${ }^{\ddagger}$ S. \\ Chenot,$\ddagger$ Y. Cordier, ${ }^{\ddagger}$ B. Damilano ${ }^{\ddagger}$ and M. Vladimirova*, $\dagger$ \\ $\dagger L 2 C$, University of Montpellier, CNRS, Montpellier, France \\ $\ddagger C R H E A$, Université Côte d'Azur, CNRS, Valbonne, France \\ E-mail: maria.vladimirova@umontpellier.fr
}

\begin{abstract}
Dipolar excitons offer a rich playground for both design of novel optoelectronic devices and fundamental many-body physics. Wide GaN/(AlGa)N quantum wells host a new and promising realization of dipolar excitons. We demonstrate the inplane confinement and cooling of these excitons, when trapped in the electrostatic potential created by semitransparent electrodes of various shapes deposited on the sample surface. This result is a prerequisite for the electrical control of the exciton densities and fluxes, as well for studies of the complex phase diagram of these dipolar bosons at low temperature.
\end{abstract}

\section{Keywords}

exciton fluid, electrostatic traps, cooling, gallium nitride

Dipolar excitons, Coulomb-bound but spatially separated electron-hole pairs, have a long life-time and a built-in dipole moment that offer an opportunity for the cooling and electrical 
control of exciton fluids. ${ }^{1-7}$ Various intriguing quantum phenomena including Bose-Einsteinlike condensation, darkening and superfluidity of excitons have been recently reported. ${ }^{8-15}$ Albeit demonstrated at very low temperatures, those phenomena are promising for better understanding of new states of matter, but also for potential applications in excitonic devices with novel functionalities. ${ }^{7}$

The recent emergence of high quality wide-bandgap semiconductor quantum wells (QWs) and two-dimensional Van der Waals heterostructures, hosting dipolar excitons with large exciton binding energies and built-in electric fields, has given a new impetus to this research. ${ }^{16-20}$ Room temperature exciton transport in GaN/(AlGa)N QWs has been demonstrated, ${ }^{17}$ as well as its electrical control in $\mathrm{MoS}_{2}-\mathrm{WSe}_{2}$ heterostructures. ${ }^{20}$ The latter is particularly important in the quest for exciton-based efficient interconnects between optical data transmission and electrical processing systems. ${ }^{7}$

However, as demonstrated in GaAs-based QWs, trapping, cooling and control of the density of dipolar excitons is essential to address various collective states, such as BoseEinstein-like condensates, that may form at low temperatures. ${ }^{12,13}$ Excitons in GaN and ZnO-based nanostructures have relatively high binding energies $\left(E_{b}\right.$ up to $100 \mathrm{meV}$ in narrow and $50 \mathrm{meV}$ in wide $\mathrm{ZnO}$ QWs, and typically 5 times less in GaN QWs $)^{21-24}$ and small Bohr radii ( $a_{B} \approx 2-3 \mathrm{~nm}$ in the bulk), ${ }^{23,24}$ as compared to GaAs-based structures (typically $4<$ $E_{b}<10 \mathrm{meV}$ in the QWs and $a_{B} \approx 10 \mathrm{~nm}$ in the bulk). ${ }^{25}$ Thus, they could exhibit quantum properties at higher temperatures. ${ }^{26}$ However, the additional challenges that must be faced to achieve such degree of control are numerous. These may include nonradiative losses, ${ }^{27,28}$ megavolt per centimeter-strong built-in electric fields, ${ }^{29}$ an exponential dependence of the lifetime on the exciton density under high excitation conditions, ${ }^{30-32}$ and the guided-andscattered light that must be distinguished from the exciton photoluminescence (PL). ${ }^{17,33}$

In this letter we overcome those challenges and report on the realization of $\sim 10 \mu \mathrm{m}$ $\times 50 \mu \mathrm{m}$-size thermalized exciton fluid, trapped in the plane of a $\mathrm{GaN} /(\mathrm{GaAl}) \mathrm{N}$ quantum well grown on a free-standing GaN substrate. Our objective is to obtain an area uniformly 
filled with excitons, with a density that can be controlled by the excitation power. To this end we fabricate electrostatic traps of various shapes by depositing semi-transparent metallic layers on top of the semiconductor nanostructure. Such strategy has already been used in GaAs-based heterostructures, ${ }^{3,34}$ but here it has to be adapted to the case of strongly polar heterostructures.

Indeed, in $\mathrm{GaN} /(\mathrm{AlGa}) \mathrm{N}$ heterostructures the built-in electric field due to spontaneous and piezoelectric polarization is of order of megavolt per centimeter. ${ }^{29,35,36}$ It pushes electrons and holes towards the two opposite interfaces of the QW. An exciton consisting of an electron and a hole spatially separated along the growth direction presents a permanent dipolar moment, roughly given by the QW width. The quantum confined Stark effect is so strong that the ground state exciton energy ( $E_{0}$ in Fig. 1 (a)) is lower (in this work by $\approx 300$ $\mathrm{meV}$ ) than that of the exciton energy in bulk GaN. The thin semitransparent metallic layer deposited on top of the structure reduces the electric field in the QW underneath, increasing exciton ground state energy: $E_{G}$ in Fig. 1 (a). Using this effect, the energy of the exciton can be modulated, making possible the on-demand patterning of the in-plane potential energy for the QW excitons. Note that this trapping scheme is opposite to the one previously developed and most frequently used in GaAs QWs, where excitons are trapped in the regions covered by the metal, where the electric field is the strongest. ${ }^{37}$

We also compare various sizes and shapes of the electrodes and show that quasi-onedimensional linear traps are optimal host structures for the exciton fluids (Fig. 1 (b)). They allow us to avoid the dilution of the exciton fluid due to radial extension, and thus to achieve high exciton densities as far as $100 \mu \mathrm{m}$ away from the excitation spot. Moreover, in this geometry, the relative contribution of the parasitic guided-and-scattered light (which is not confined by the electrodes so that its intensity decreases as $1 / r$, where $r$ is the distance from the excitation spot) is substantially reduced with respect to the confined excitons emission. Finally, implementation of quasi-one-dimensional zigzag-shaped trap (Fig. 1 (c)) provides unambiguous evidence of the diffusive transport of the trapped excitons. 

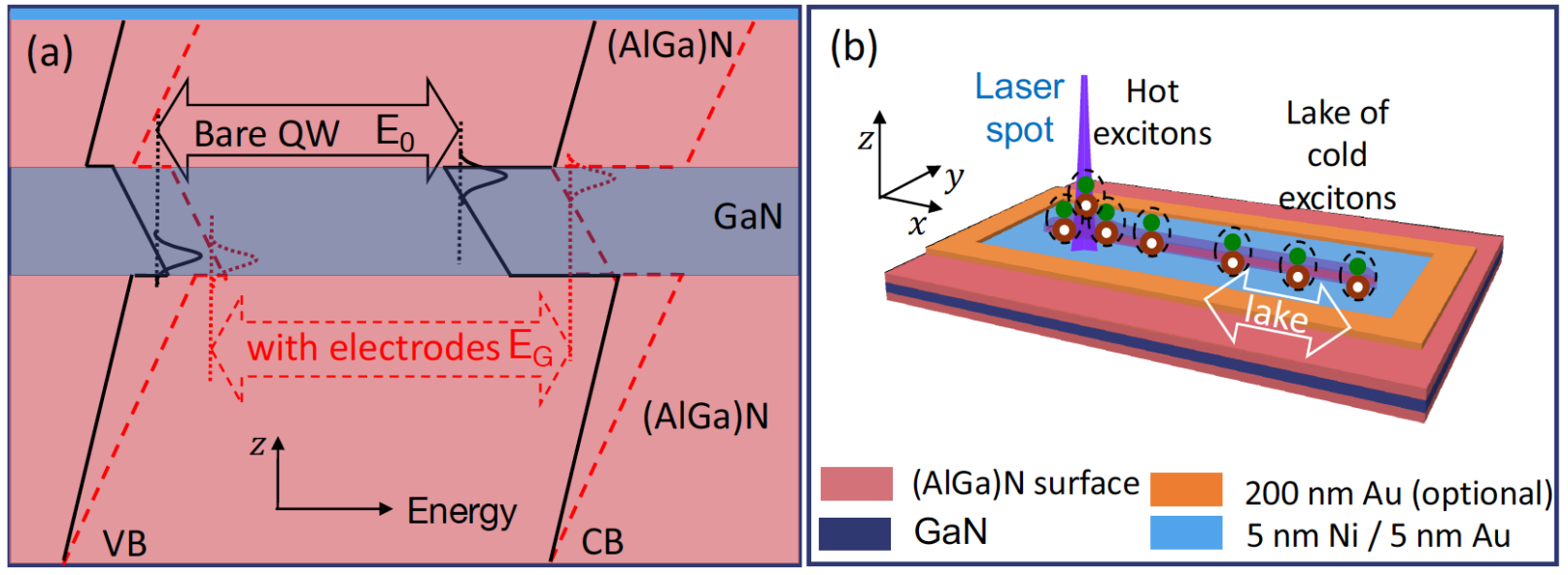

(c) $D=40 \& 80 \mu \mathrm{m}$
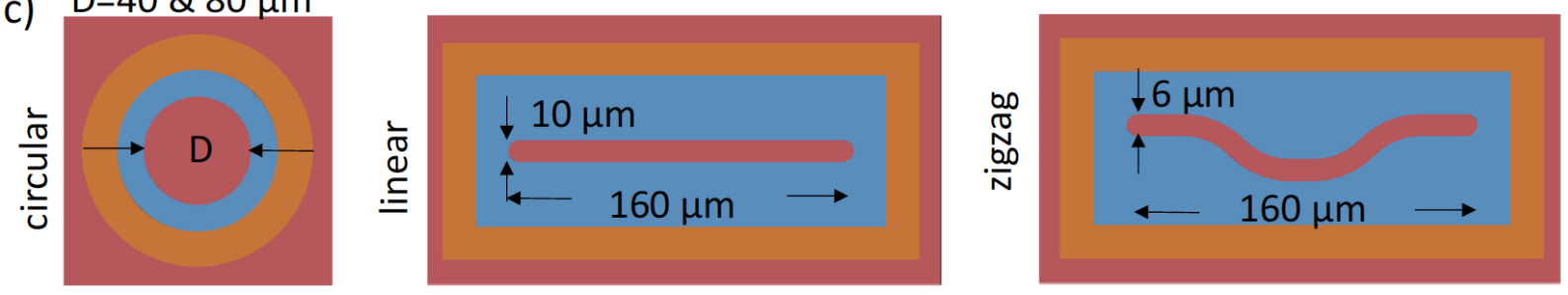

Figure 1: (a) Band diagram of the GaN/(AlGa)N QW in the absence (black solid line) and in the presence of the top electrodes (red dashed line). Corresponding carrier wavefunctions, energy levels, and interband transition energies are shown with the same color code. (b) Artist view of the studied structure. (c) The three types of traps under study. 


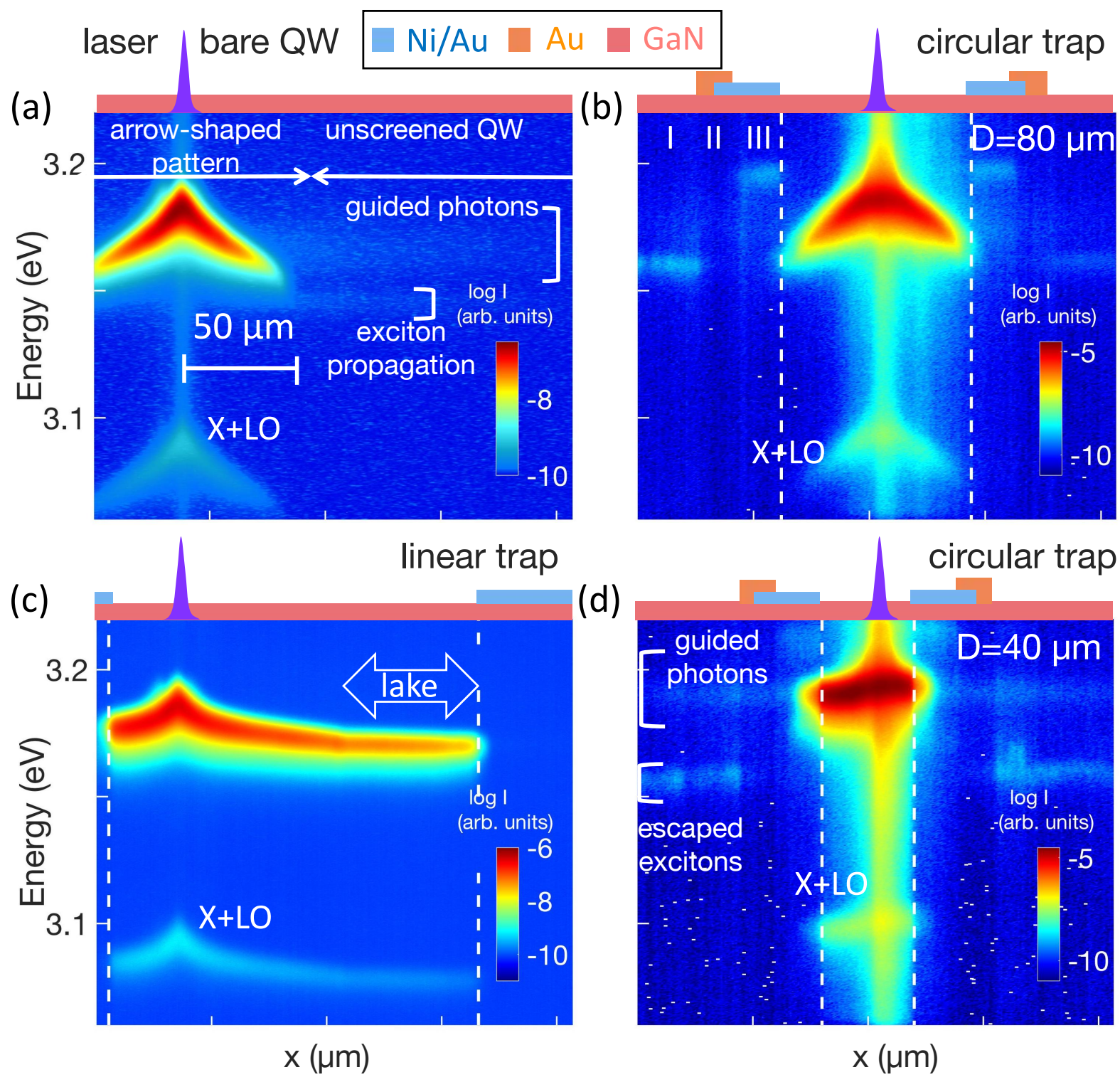

Figure 2: PL intensity (color-encoded in log scale) measured at $T=4 \mathrm{~K}$ under point-like excitation of a bare QW (a) and of the QW patterned with electrodes providing circular (b), (d) and linear (c) traps for the excitons. The electrode patterns and the laser spot position are shown schematically. Excitation power densities are $P=1 \mathrm{~mW} / \mathrm{cm}^{2}$ (a), $P=500 \mu \mathrm{W} / \mathrm{cm}^{2}$ (b, d) and $P=850 \mu \mathrm{W} / \mathrm{cm}^{2}$ (c). The emission is spatially resolved along $x$-axis, excitation spot is positioned in the center of the trap along $y$-axis. 
We investigate a 7.8 nm-wide GaN QW sandwiched between 50 (top) and $100 \mathrm{~nm}$ wide (bottom) $\mathrm{Al}_{0.11} \mathrm{Ga}_{0.89} \mathrm{~N}$ barriers, grown on a free-standing GaN substrate (LUMILOG, threading dislocation density $\left.2 \times 10^{7} \mathrm{~cm}^{-2}\right)$. The exciton radiative lifetime in such structure (in the zero zero-density limit) is estimated as $\tau_{0} \approx 10 \mu \mathrm{s},{ }^{17}$ exciton binding energy as $E_{b}=10 \mathrm{meV}$ and the built-in electric field $F=980 \mathrm{kV} / \mathrm{cm}$. The semi-transparent electrodes consisting of $5 \mathrm{~nm}$ of $\mathrm{Au}$ on top of $5 \mathrm{~nm}$ of $\mathrm{Ni}$ are evaporated on the sample surface and patterned by optical lithography. These electrodes ensure $\approx 1 \mathrm{eV}$ Schottky barrier shift ${ }^{38-40}$ and are used in state-of-art GaN/(Al,Ga)N high electron mobility transistors. ${ }^{41}$ Some of the patterns are additionally covered by a $200 \mathrm{~nm}$-thick Au layer for subsequent wire bonding (Fig. 1 (b)). This layer is opaque. Three electrode geometries used to create in-plane traps are illustrated in Fig. 1 (c): circular trap, linear trap, and zigzag-shaped trap. In contrast with GaAs-based devices, in the absence of the applied bias the regions with bare surface are those where the electric field is the strongest, and where excitons accumulate due to maximized confinement, while under the electrodes the field is lower. The details on the calculation of the band profiles and the confinement energies using coupled Schrödinger and Poisson equations are provided in Supporting Information (SI). These calculations allow us to estimate a trapping potential energy $E_{t}=E_{G}-E_{0} \approx 50 \mathrm{meV}$, in reasonable agreement with experimental results, as shown below. Here $E_{0}$ and $E_{G}$ are the exciton energies in the bare QW and in the QW covered by the electrodes, respectively, see Fig. 1 (a).

In most of our experiments the sample is cooled down to $4 \mathrm{~K}$ and the QW excitons are created within a $1 \mu \mathrm{m}$-diameter spot on the sample surface. We use either continuous wave (CW) optical pumping at $\lambda=266 \mathrm{~nm}$, or quasi-CW pumping at $360 \mathrm{~nm}$ (picosecond pulses at $80 \mathrm{MHz}$ frequency, much higher than inverse exciton lifetime $\left.1 / \tau_{0} \approx 0.1 \mathrm{MHz}\right)$. The PL spectra are acquired by a charge coupled device (CCD) camera with $\approx 1 \mu \mathrm{m}$ spatial resolution. More details can be found in Fedichkin et al. ${ }^{17}$ and in SI.

The exciton transport and trapping, as well as their dependence on the trap geometry, are presented in Fig. 2. It shows color-encoded PL spectra, measured at various distances from 
the excitation spot along the $x$-axis ( $c f$ Fig. $1(\mathrm{~b})$ ). The emission from the bare surface (Fig. 2 (a), no trapping electrodes) is compared to the circular traps with diameters $D=80 \mu \mathrm{m}$ and $D=40 \mu \mathrm{m}$ (Fig. 2 (b), (d), circular traps) and quasi-one-dimensional channel ( Fig. 2 (c), linear trap). Apart from the broad emission directly under the excitation point, at any distance from the spot the spectrum exhibits two main peaks: the zero-phonon exciton emission line and the first phonon replica $91 \mathrm{meV}$ below $(\mathrm{X}+\mathrm{LO}) .{ }^{42}$ The energy, intensity, and linewidth of the excitonic PL decrease when moving away from the excitation position. These effects are due to the screening of the electric field along the growth direction induced by the photocreated carriers: the highest density under the pumping spot corresponds to the maximum screening (lowest electric field), and thus to the highest emission intensity and energy. ${ }^{17,30,33}$ The pump-induced emission energy shift is referred to as the blue shift, $E_{B S}$. Its magnitude roughly corresponds to the repulsive interaction between the dipolar excitons. $E_{B S}$ can be used for estimating the exciton density as $n=E_{B S} / \phi_{0}$, where $\phi_{0}=$ $1.50 \times 10^{-13} \mathrm{eV} \cdot \mathrm{cm}^{2}$ is the coefficient extracted from the self-consistent solution of the Schrödinger and Poisson equations (see also SI). ${ }^{17,30,33}$ This estimate could be improved taking into account excitonic correlations (see discussion below). ${ }^{26,43,44}$

Let us compare the spatial profiles of the emission from the bare QW and circular traps. In the bare sample (Fig. $2(\mathrm{a}))$ an arrow-shaped pattern with $\approx 50 \mu \mathrm{m}$-width is accompanied by weak emission $\approx 50 \mu \mathrm{m}$ away from the spot at an approximately constant energy of $3.145 \mathrm{eV}$ (this emission is due to small fraction of excitons that propagated diffusively) and broad emission band at $3.16-3.18 \mathrm{eV}$ (this light is emitted by the excitons under the spot, guided along the sample plane, and then scattered out of the sample by surface roughness). The same emission pattern has been previously observed in similar samples. ${ }^{17}$ In the sample with $80 \mu \mathrm{m}$ circular trap an arrow-shaped pattern of similar size can be identified. This means that we load the trap with excitons, but the density across the trap is not homogeneous. By contrast, in a smaller trap, the arrow-shaped pattern is replaced by a flat pattern. This suggests that the in-plane potential is completely screened by the trapped excitons, or, in 


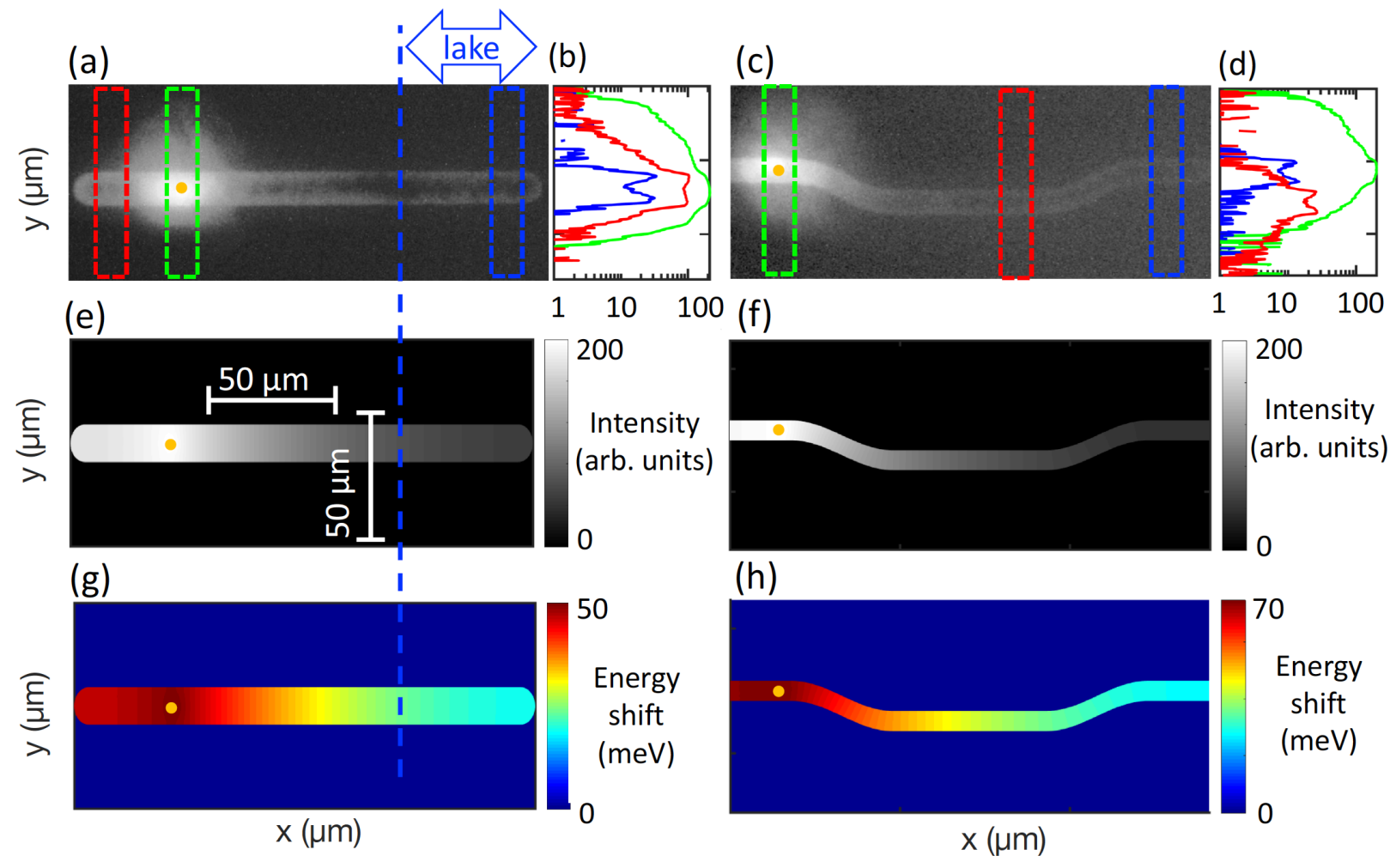

Figure 3: Grey scale-encoded $x y$ map of the spectrally-integrated $(3.05-3.25 \mathrm{eV})$ PL intensity in the linear (a) and zigzag-shaped (c) traps at $P=50 \mathrm{~mW} / \mathrm{cm}^{2}$. Yellow dots indicate the laser spot position. The intensity profiles shown (b) for linear trap and (d) for zigzag trap correspond to intensities integrated over $x$, in the windows displayed by dashed lines of the same color. (e-h) are the results of the drift-diffusion modeling. Both intensity (e-f) and energy shift $E_{B S}(\mathrm{~g}-\mathrm{h})$ are shown. Blue dashed lines delimit the part of the linear trap denoted as lake, where the variations of the PL intensity and energy (and thus exciton density) are weak. The intensity scales in (a), (c) and (e-f) are identical. 
other words, that the trap is fully filled.

In both circular traps we observe weak, but clearly patterned emission from the outer regions of the trap. Three different zones can by distinguished outside the trap: bare QW (I), thick gold electrode (II) and semitransparent electrode (III). The emission from the zone I is centered at the energy $E_{0}$ of the empty QW. The excitons that emit this light have escaped from the trap and propagate diffusively, their density is small enough to produce no measurable blue shift. This emission is alike the emission in a bare sample more than $60 \mu \mathrm{m}$ away from the spot, where the density of the photocreated carriers is low, and the built-in electric field is not screened. The small difference in the energy between the bare sample $(\approx 3.15 \mathrm{eV}$ in panel $(\mathrm{a}))$ and the "outside the trap" zone I $(\approx 3.16 \mathrm{eV}$ in panels $(\mathrm{b})$ and $(\mathrm{d}))$ could be due to electrostatic effects induced by closely lying electrodes. In the smaller circular trap one can also see in zone I an emission at the same energy as the strongest emission at the center of the trap $(\approx 3.18 \mathrm{eV})$. It is alike broad emission in bare sample at more than $50-60 \mu \mathrm{m}$ from the spot. This light is emitted by the excitons in the trap, guided along the sample plane over tens of microns, and then scattered out of the sample by surface roughness. Finally, the emission in zone III, the closest to the trap, provides an estimation of the trap depth. This is particularly clear for the $80 \mu \mathrm{m}$-wide trap. A few excitons that escaped from the trap in the region covered by the semitransparent electrodes emit light at $E_{G}=3.19 \mathrm{eV}$. This corresponds to the modulation of the confinement potential energy induced by the metallic gates. The measured trapping potential energy $E_{t}=E_{G}-E_{0} \sim 30 \mathrm{meV}$ is slightly below $50 \mathrm{meV}$ expected from the model based on the solution of the coupled Schrödinger and Poisson equations (see SI).

The advantage of the linear trap with respect to the circular one is quite natural: it allows us to eliminate the radial dilution of the exciton density ( $\propto 1 /$ distance), and thus to keep relatively high densities of excitons at significant distances from the excitation spot. Moreover, in circular traps, as well as in the bare QW, the exciton emission competes with the guided-and-scattered light (although these two kinds of emissions can be spectrally dis- 
tinguished). By contrast, in the linear traps the guided-and-scattered light is subject to radial dilution, while the excitons are confined in the trap, which is quasi-one-dimensional.

The linear trap (Fig. 2 (c)) exhibits a particularly remarkable emission pattern. While close to the excitation spot one can still distinguish an arrow-shaped pattern, starting from $\approx 50 \mu \mathrm{m}$ and further away the emission energy and intensity are almost independent of the position. This suggests that we have created, within the trap, an area homogeneously filled with carriers, that will be referred to as an exciton lake. At $P=850 \mu \mathrm{W} / \mathrm{cm}^{2}$ used in Fig. 2 (c), the blue shift of the exciton energy in the lake does not exceed $20 \mathrm{meV}$. This is less than the trapping potential energy, making possible the accumulation of the excitons in the trap.

More insight into exciton in-plane propagation and trapping can be obtained from the spectrally integrated emission intensity maps shown in Fig. 3 (a) and (c) for linear and zigzag-shaped traps, respectively. These images are acquired through a CMOS camera after a spectral filter matching the energy range shown in Fig. 2. Two main features can be distinguished in these images. First, along the $y$-direction the emission from the traps is inhomogeneous. The emission from the borders is stronger than the one from the center of the trap. This is additionally illustrated in Fig. 3 (b) and (d), where we show the profiles taken under the spot (green line) and at two other positions along the $x$-direction (red and blue lines) for linear and zigzag-shaped traps, respectively. The observed inhomogeneity is probably related to the shape of the trap, suggesting that the electric field in $z$-direction varies progressively between the in-plane regions covered by the metal and the regions with bare surface.

Second, under the pumping power density used in this particular experiment $\left(50 \mathrm{~mW} / \mathrm{cm}^{2}\right)$ the traps are strongly leaking. Indeed, the laser spot (position indicated by the yellow dot) is surrounded by a circular halo, suggesting that under the spot the carrier density is high enough to screen the trapping potential energy $E_{t} \approx 30 \mathrm{meV}$. This halo being radially symmetric, it may include contribution from the photon diffusion, analogous to the one in Fig. 
2 (a). However, the in-plane profiles of the emission in the remote regions of the traps are inconsistent with the radial symmetry of the light propagation. This is particularly obvious from the zigzag-shaped trap emission. Thus, these experiments prove unambiguously that the emission as far as $100 \mu \mathrm{m}$ away from the excitation spot results from the exciton transport, rather than from photon diffusion.

To go further and quantify relevant parameters of the exciton propagation, we apply the exciton transport model developed in Fedichkin et al. ${ }^{17}$, Rapaport et al. ${ }^{45}$ to the case of transport in a two-dimensional trapping potential. For the sake of simplicity, we neglect some experimentally observed features: we assume infinite trap barriers (no leakage) and spatially homogeneous potential within the trap. The exciton transport is assumed to be limited by the presence of randomly distributed scatterers (Model C of Fedichkin et al. ${ }^{17}$ ) so that the diffusion coefficient is given by $D=\ell \sqrt{2 k_{B} T / M_{X}^{\perp}}$, where $M_{X}^{\perp}=1.8 m_{0}$ is the in-plane exciton mass, $k_{B}$ is the Boltzmann constant, $m_{0}$ is the free electron mass, and $\ell=10 \mathrm{~nm}$ is the exciton scattering length. The latter, together with the fraction of the photons absorbed in the QW $\alpha=10^{-4}$ are the only fitting parameters. The details on the transport modeling are given in the SI. The results of the numerical solution of the diffusion equation are shown in Fig. 3 (e-h) for both linear (e, g) and zigzag-shaped (f, h) traps. The simulation reproduces the propagation and filling of the traps, both intensity and energy profiles as well as the formation of the region $\approx 100 \mu \mathrm{m}$ away from the laser spot, where exciton emission energy and intensity are almost constant: the exciton lake.

In the following we analyze the spectral properties of the emission from the lake region in the linear trap as a function of excitation power density, in order to demonstrate density and temperature control of the dipolar excitons. Figure 4 (a) shows a set of exciton emission spectra (solid lines) averaged over the lake region of the linear trap (white arrow in Fig. 2 (c)). The power density varies from 50 to $500 \mu \mathrm{W} / \mathrm{cm}^{2}$ and sample temperature is fixed at $T=4 \mathrm{~K}$. One can identify a blue shift of the emission line $(\approx 20 \mathrm{meV})$ and increasing intensity that accompanies the increase of the power. The line shape is not Gaussian, as 


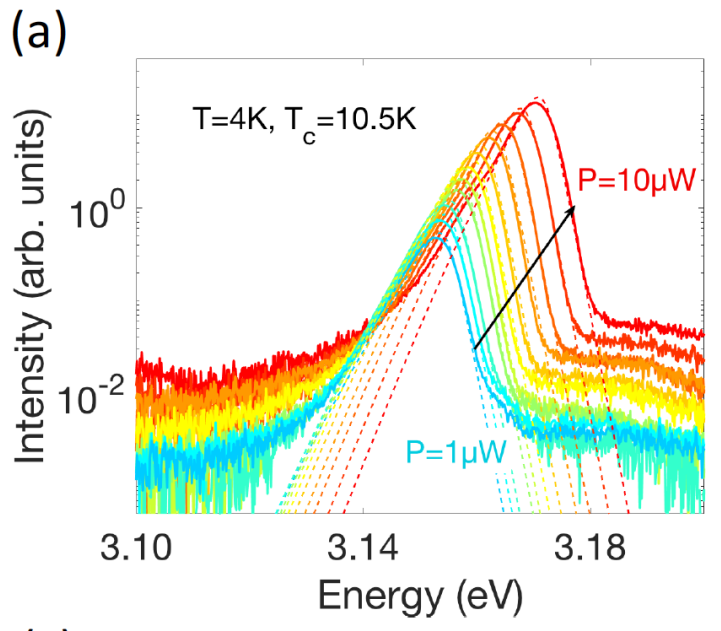

(c)

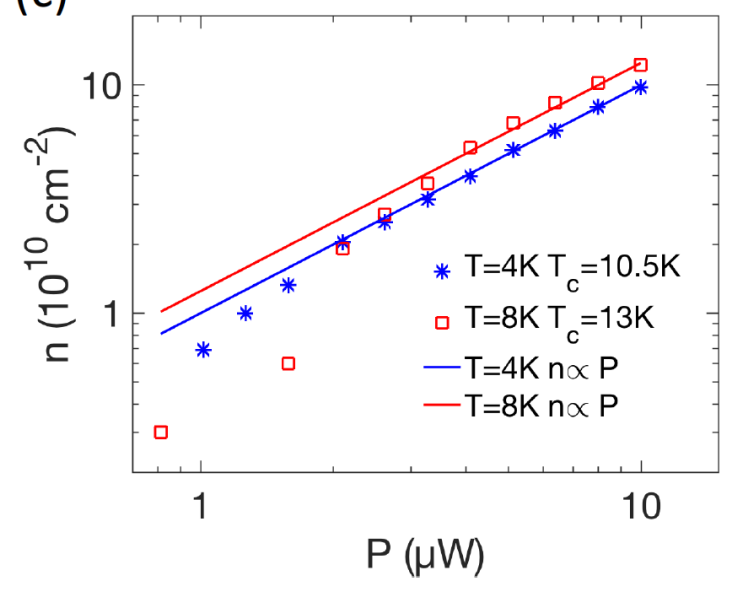

(b)

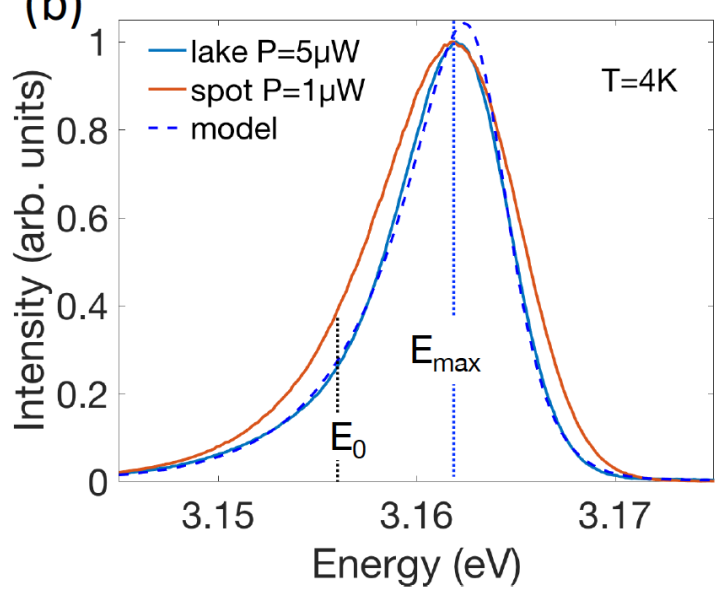

(d)

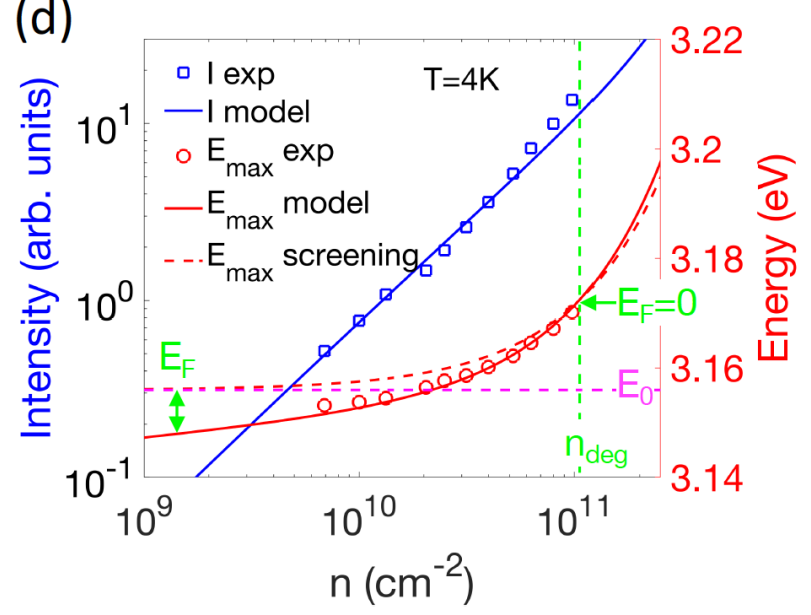

Figure 4: (a) Exciton emission spectra integrated over the lake area at different powers (solid lines) and the results of the fitting procedure with power-independent carrier temperature $T_{c}$ (dashed lines). (b) Comparison between the normalized spectra in the lake at $P=$ $250 \mu \mathrm{W} / \mathrm{cm}^{2}$ and under the laser spot at $P=50 \mu \mathrm{W} / \mathrm{cm}^{2}$ Vertical lines point the energies $E_{0}$ of the empty QW and $E_{\max }$ corresponding to the intensity peak at a given power. (c) Exciton density as a function of the power extracted from the modeling of the spectra at $4 \mathrm{~K}$ with $T_{c}=10.5 \mathrm{~K}$ shown in (a) and at $8 \mathrm{~K}$ with $T_{c}=13.5 \mathrm{~K}$ (spectra shown in $\mathrm{SI}$ ). Solid lines are guides for the eyes indicating linear slope. (d) Integrated intensity (left scale) and the spectral peak energy $E_{\max }$ (right scale) as a function of the carrier density extracted from the data (symbols) and the model (solid lines) shown in (a). The dashed lines show the density corresponding to the degeneracy condition (green), the energy of the empty QW $E_{0}$ (magenta) and the calculated variation of the QW energy $E_{\max }$ due to carrier-induced screening of the built-in electric field (red). 
one could expect for the emission dominated by inhomogeneous broadening. Instead, an exponential shape of both high- and low-energy tails is apparent. Similar profiles have already been observed in PL spectra of dipolar excitons. ${ }^{10,46}$ In particular, Schinner et al use Fermi-Dirac distribution as a cutoff function, to account for the thermal smearing of the filling of the trap with the dipolar exciton liquid. ${ }^{10}$ An argument that was put forward in favor of this approach is that in the case of the bosonic dipolar liquid, the strong spatial dipolar repulsion plays the role of the Pauli principle in the Fermi-Dirac distribution. Note however, that more elaborate theory accounting properly for the bosonic nature of dipolar excitons is needed for more precise description of our experiments. To account for the inhomogeneous broadening and describe the low-energy tail of the spectrum, Schinner et al multiply the Fermi-Dirac distribution by a phenomenological Gaussian function. ${ }^{10}$ In our samples the low-energy tail of the excitonic emission is rather exponential, similar to Stern et al. ${ }^{46}$ Therefore we model this peculiar shape with the product of the exponential function (to describe the low-energy tail of the spectrum) and the Fermi-Dirac distribution (to account for the temperature-broadened steep decrease of the high-energy tail of the spectrum). The details on the model and fitting procedure are given in SI.

The efficiency of the exciton cooling in the lake can be estimated by fitting the set of data at different powers, as shown in Fig. 4 (a). From such a procedure, we extract the following fitting parameters: the zero-density exciton energy $E_{0}=3.156 \mathrm{eV}$, the carrier densities $n$, and the carrier temperature $T_{c}=10.5 \mathrm{~K}$. The latter appears to be power independent, albeit different from the nominal sample temperature $T=4 \mathrm{~K}$. The resulting carrier densities are shown in Fig. 4 (c) as a function of the laser power for two sets of data at different nominal temperatures. For $T=4 \mathrm{~K}$ we get $T_{c}=10.5 \mathrm{~K}$ and for $T=8 \mathrm{~K}$ we get $T_{c}=13.5 \mathrm{~K}$. We speculate that the difference between carrier temperature and the nominal lattice temperature results from the poor thermalization of the crystal lattice in the cold finger cryostat, and the real lattice temperature is higher, so that $T \approx T_{c}$. Apart from the very lowest powers $(P<2 \mu \mathrm{W}$, where nonradiative recombination may dominate over 
the radiative one), the carrier density increases linearly with power, indicating vanishing contribution of nonradiative losses. The higher density at higher temperature is a signature of the increasing exciton lifetime in the QW. ${ }^{47,48}$

It is instructive to compare the emission peak with a given energy measured in the lake $(P=5 \mu \mathrm{W})$, and directly at the excitation spot, but at lower power, $P=1 \mu \mathrm{W}$. Remarkably, even at a factor of 5 lower excitation power, the emission under the spot is broader than the one in the lake and does not have the same profile, so that the fitting procedure used for the lake spectra is not viable. This suggests that the impact of optical excitation goes beyond the heating of the exciton gas and the screening of the built-in electric field, that are both taken into account by the model.

The determination of the carrier density in the nanostructures hosting dipolar excitons is a very delicate question since it relies on the knowledge of the exciton-exciton interactions. The values of $n$ given in Fig. 4 (c-d) are obtained in the rough semi-phenomenological approximation neglecting excitonic correlations (see above and SI). The same approximation was used in Refs., ${ }^{17,33}$ and it is conceptually similar to the plate capacitor model. ${ }^{49,50}$ The effect of the exciton-exciton interactions was investigated theoretically and experimentally in Refs. ${ }^{43,44,51-53}$ and results in a density and temperature-dependent correction factor that leads to the underestimation of the density by up to one order of magnitude at the lowest powers and temperatures. Nevertheless, it is not straightforward to apply those considerations to our system, and a more advanced analysis of the data remains to be done. In this work we determine the lower limit for the carrier density, and bear in mind that excitonic correlations may increase it by up to a factor of 10 .

Fig. 4 (d) summarizes the results of the modeling. Both energy (red circles, right scale) and integrated intensity (blue circles, left scale) of the excitonic emission are shown as a function of the exciton density extracted from the model (solid lines). This representation allows us to appreciate the role of the two density-induced contributions to the shift of the emission energy: the screening of the built-in electric field $\left(E_{0}+\phi_{0} n\right.$, red dashed line) 
and the phase space filling that leads to an additional shift of the emission that can be described in terms of the variation of the Fermi energy $E_{F}=E_{\max }-E_{0}+\phi_{0} n$. The latter appears in Fig. $4(\mathrm{~d})$ as the difference between the red solid line, showing the full model and red dashed line, showing the screening effect (三 energy blue shift) on the emission energy. The negative Fermi energy corresponds to the situation were the energy $E_{\max }$ of the PL peak lies below the band energy determined by the screening effect $E_{0}+\phi_{0} n$, so that optically created electron (hole) gas is non-degenerate $\left(n<n_{\text {deg }}\right)$. One can see that the Fermi contribution is not negligible at low densities, at least within the approximations described above. Importantly, even at maximum carrier densities achieved in the lake the carriers remain in the non-degenerate limit $n<n_{\text {deg }} \approx 10^{11} \mathrm{~cm}^{-2}$ ( corresponding to $\left.E_{F}<0\right)$. This range of densities is well below excitonic Mott transition expected to occur progressively above $n_{M o t t}=6 \times 10^{11} \mathrm{~cm}^{-2}$, so that excitons are expected to form within the electronhole liquid. ${ }^{54}$ Moreover, the integrated emission intensity is well described by the model, and it increases almost linearly with the exciton density, at least up to $P=5 \mu \mathrm{W}$. This means that the carrier densities are low enough, so that exponential enhancement of the radiative emission rate remains negligible, in contrast with what is usually observed in polar heterostructures at higher carrier densities. ${ }^{16,17,30-33}$

In conclusion, we have shown that dipolar excitons can be efficiently trapped in the plane of $\mathrm{GaN} /(\mathrm{AlGa}) \mathrm{N}$ QWs. In previous works on GaN QWs hosting dipolar excitons, the mutual repulsion of excitons led to a fast radial expansion and dilution of the exciton gas, accompanied by dramatic variation of the exciton energy and lifetime. ${ }^{17,33}$ Here we overcome these problems, and create spatial areas (referred to as lakes) with tens of micrometers characteristic size, homogeneously filled with excitons with well-defined emission energy and lifetime. We show that the exciton fluid trapped in the lake is at macroscopic thermodynamical equilibrium with well-defined temperature close to the sample temperature. The density of this cold exciton fluid can be controlled by changing the laser excitation power.

The realization of traps relies on the deposition of metallic gates on the surface. Both 
the electrode shape, and the characteristic electric fields that must be manipulated (of order of $F=1 \mathrm{MV} / \mathrm{cm}$ ) are quite different from the known GaAs-based nanostructures. Such strong built-in electric field in nitride-based structures could be not only a challenge to meet, but also an opportunity to avoid spurious photocurrents that limit exciton mobility and coherence properties. These photocurrents are not easy to avoid in other materials hosting dipolar excitons (either GaAs-based or Van der Waals heterostructures), where a voltage is usually applied across the regions where excitons accumulate.

The present results could be transposed and repeated in $\mathrm{ZnO}$, an other wide band gap semiconductor with higher exciton binding energy. This make them potentially promising for the realization of exciton-based optoelectronic devices operating at room temperature. Our results pave the way towards such functionalities, although the electrical control of the exciton fluxes remains to be demonstrated. Finally, the detailed spectroscopic study of dipolar exciton fluids at various densities made possible by the presented results, may shed new light on their intriguing properties, such as macroscopic coherence and condensation, predicted to show up in GaN QWs at higher temperatures than in GaAs.

\section{Acknowledgement}

The authors thank D. Scalbert and M. Leroux for enlightening discussions. This work was supported by the French National Research Agency via ANR OBELIX (Grant No. No ANR-15-CE30-0020-02) and LABEX GANEX (Grant No. ANR-11-LABX-0014). GANEX belongs to the public funded "Investissements d'Avenir" program managed by the French National Research Agency.

\section{Supporting Information Available}

Sample; Experimental methods; Modeling the confinement potential, transition energies and carrier lifetimes; Drift - diffusion modeling of the exciton transport; Modeling of the PL 
spectra, estimation of the carrier density.

\section{References}

(1) Yudson, V. I.; Lozovik, Y. E. A new mechanism for superconductivity: pairing between spatially separated electrons and holes. Soviet Physics JETP 1976, 44, 389.

(2) Miller, D. A. B.; Chemla, D. S.; Damen, T. C.; Gossard, A. C.; Wiegmann, W.; Wood, T. H.; Burrus, C. A. Electric-Field Dependence of Optical-Absorption Near the Band-Gap of Quantum-Well Structures. Phys. Rev. B 1985, 32, 1043-1060.

(3) Huber, T.; Zrenner, A.; Wegscheider, W.; Bichler, M. Electrostatic Exciton Traps. physica status solidi (a) 1998, 166, R5-R6.

(4) Ivanov, A. L.; Littlewood, P. B.; Haug, H. Bose-Einstein statistics in thermalization and photoluminescence of quantum-well excitons. Phys. Rev. B 1999, 59, 5032-5048.

(5) Butov, L. V.; Shashkin, A. A.; Dolgopolov, V. T.; Campman, K. L.; Gossard, A. C. Magneto-optics of the spatially separated electron and hole layers in $\mathrm{GaAs} / \mathrm{Al}_{x} \mathrm{Ga}_{1-x} \mathrm{As}$ coupled quantum wells. Phys. Rev. B 1999, 60, 8753-8758.

(6) High, A. A.; Novitskaya, E. E.; Butov, L. V.; Hanson, M.; Gossard, A. C. Control of exciton fluxes in an excitonic integrated circuit. Science 2008, 321, 229-231.

(7) Butov, L. V. Excitonic devices. Superlattices and Microstructures 2017, 108, 2-26.

(8) High, A. A.; Leonard, J. R.; Hammack, A. T.; Fogler, M. M.; Butov, L. V.; Kavokin, A. V.; Campman, K. L.; Gossard, A. C. Spontaneous coherence in a cold exciton gas. Nature 2012, 483, 584-588.

(9) Shilo, Y.; Cohen, K.; Laikhtman, B.; West, K.; Pfeiffer, L.; Rapaport, R. Particle correlations and evidence for dark state condensation in a cold dipolar exciton fluid. Nature Communications 2013, 4, 2335. 
(10) Schinner, G. J.; Repp, J.; Schubert, E.; Rai, A. K.; Reuter, D.; Wieck, A. D.; Govorov, A. O.; Holleitner, A. W.; Kotthaus, J. P. Many-body correlations of electrostatically trapped dipolar excitons. Phys. Rev. B 2013, 87, 205302.

(11) Cohen, K.; Shilo, Y.; West, K.; Pfeiffer, L.; Rapaport, R. Dark High Density Dipolar Liquid of Excitons. Nano Lett. 2016, 16, 3726-3731.

(12) Butov, L. V. Collective phenomena in cold indirect excitons. J. Exp. Theor. Phys. 2016, $149,505$.

(13) Combescot, M.; Combescot, R.; Dubin, F. Bose-Einstein condensation and indirect excitons: a review. Rep. Prog. Phys. 2017, 80, 066501.

(14) Anankine, R.; Beian, M.; Dang, S.; Alloing, M.; Cambril, E.; Merghem, K.; Carbonell, C. G.; Lemaître, A.; Dubin, F. Quantized Vortices and Four-Component Superfluidity of Semiconductor Excitons. Phys. Rev. Lett. 2017, 118, 127402.

(15) Misra, S.; Stern, M.; Joshua, A.; Umansky, V.; Bar-Joseph, I. Experimental Study of the Exciton Gas-Liquid Transition in Coupled Quantum Wells. Phys. Rev. Lett. 2018, 120, 047402.

(16) Kuznetsova, Y. Y.; Fedichkin, F.; Andreakou, P.; Calman, E. V.; Butov, L. V.; Lefebvre, P.; Bretagnon, T.; Guillet, T.; Vladimirova, M.; Morhain, C.; Chauveau, J. M. Transport of indirect excitons in $\mathrm{ZnO}$ quantum wells. Opt. Lett., 2015, 40, 3667-3670.

(17) Fedichkin, F.; Andreakou, P.; Jouault, B.; Vladimirova, M.; Guillet, T.; Brimont, C.; Valvin, P.; Bretagnon, T.; Dussaigne, A.; Grandjean, N.; Lefebvre, P. Transport of dipolar excitons in (Al,Ga)N/GaN quantum wells. Phys. Rev. B 2015, 91, 205424.

(18) Rivera, P.; Schaibley, J. R.; Jones, A. M.; Ross, J. S.; Wu, S.; Aivazian, G.; Klement, P.; Seyler, K.; Clark, G.; Ghimire, N. J.; Yan, J.; Mandrus, D. G.; Yao, W.; Xu, X. Ob- 
servation of long-lived interlayer excitons in monolayer $\mathrm{MoSe}_{2}-\mathrm{WSe}_{2}$ heterostructures. Nature Communications 2015, 6, 6242.

(19) Ross, J. S.; Rivera, P.; Schaibley, J.; Lee-Wong, E.; Yu, H.; Taniguchi, T.; Watanabe, K.; Yan, J.; Mandrus, D.; Cobden, D.; Yao, W.; Xu, X. Interlayer Exciton Optoelectronics in a 2D Heterostructure p-n Junction. Nano Lett. 2017, 17, 638-643.

(20) Unuchek, D.; Ciarrocchi, A.; Avsar, A.; Watanabe, K.; Taniguchi, T.; Kis, A. Roomtemperature electrical control of exciton flux in a van der Waals heterostructure. Nature 2018, 560, 340-344.

(21) Coli, G.; Bajaj, K. K. Excitonic transitions in ZnO/MgZnO quantum well heterostructures. Appl. Phys. Lett. 2001, 78, 2861-2863.

(22) Hong, J. S.; Ryu, S. W.; Hong, W. P.; Kim, J. J.; Kim, H. M.; Park, S. H. Exciton binding energies in wurtzite $\mathrm{ZnO} / \mathrm{MgZnO}$ quantum wells. 2006 IEEE Nanotechnology Materials and Devices Conference. 2006; pp 324-325.

(23) Bigenwald, P.; Lefebvre, P.; Bretagnon, T.; Gil, B. Confined Excitons in GaN-AlGaN Quantum Wells. Physica Status Solidi (b) 1999, 216, 371-374.

(24) Gil, B. III-Nitride Semiconductors and their Modern Devices; Springer, 2014.

(25) Khramtsov, E. S.; Belov, P. A.; Grigoryev, P. S.; Ignatiev, I. V.; Verbin, S. Y.; Efimov, Y. P.; Eliseev, S. A.; Lovtcius, V. A.; Petrov, V. V.; Yakovlev, S. L. Radiative decay rate of excitons in square quantum wells: Microscopic modeling and experiment. J. Appl. Phys. 2016, 119, 184301.

(26) Laikhtman, B.; Rapaport, R. Exciton correlations in coupled quantum wells and their luminescence blue shift. Phys. Rev. B 2009, 80, 195313-12.

(27) Shockley, W.; W T Read, J. Statistics of the Recombinations of Holes and Electrons. Phys. Rev. 1952, 87, 835-842. 
(28) Hall, R. N. Electron-Hole Recombination in Germanium. physical review 1952, 87, $387-387$.

(29) Grandjean, N.; Damilano, B.; Dalmasso, S.; Leroux, M.; Laügt, M.; Massies, J. Built-in electric-field effects in wurtzite AlGaN/GaN quantum wells. J. Appl. Phys. 1999, 86, 3714 .

(30) Lefebvre, P.; Kalliakos, S.; Bretagnon, T.; Valvin, P.; Taliercio, T.; Gil, B.; Grandjean, N.; Massies, J. Observation and modeling of the time-dependent descreening of internal electric field in a wurtzite $\mathrm{GaN} / \mathrm{Al}_{0.15} \mathrm{Ga}_{0.85} \mathrm{~N}$ quantum well after high photoexcitation. Phys. Rev. B 2004, 69, 035307.

(31) Liu, W.; Butté, R.; Dussaigne, A.; Grandjean, N.; Deveaud, B.; Jacopin, G. Carrierdensity-dependent recombination dynamics of excitons and electron-hole plasma in mplane InGaN/GaN quantum wells. Phys. Rev. B 2016, 94, 195411.

(32) Chen, W.; Wen, X.; Yang, J.; Latzel, M.; Patterson, R.; Huang, S.; Shrestha, S.; Jia, B.; Moss, D. J.; Christiansen, S.; Conibeer, G. Free charges versus excitons: photoluminescence investigation of InGaN/GaN multiple quantum well nanorods and their planar counterparts. Nanoscale 2018, 10, 5358-5365.

(33) Fedichkin, F.; Guillet, T.; Valvin, P.; Jouault, B.; Brimont, C.; Bretagnon, T.; Lahourcade, L.; Grandjean, N.; Lefebvre, P.; Vladimirova, M. Room-Temperature Transport of Indirect Excitons in (Al,Ga)N/GaN Quantum Wells. Phys. Rev. Applied 2016, 6, 014011.

(34) Hammack, A. T.; Gippius, N. A.; Yang, S.; Andreev, G. O.; Butov, L. V.; Hanson, M.; Gossard, A. C. Excitons in electrostatic traps. J. Appl. Phys. 2006, 99, 066104.

(35) Bernardini, F.; Fiorentini, V. Macroscopic polarization and band offsets at nitride heterojunctions. Phys. Rev. B 1998, 57, R9427-R9430. 
(36) Leroux, M.; Grandjean, N.; Laügt, M.; Massies, J.; Gil, B.; Lefebvre, P.; Bigenwald, P. Quantum confined Stark effect due to built-in internal polarization fields in (Al,Ga)N/GaN quantum wells. Phys. Rev. B 1998, 58, R13371-R13374.

(37) Grosso, G.; Graves, J.; Hammack, A. T.; High, A. A.; Butov, L. V.; Hanson, M.; Gossard, A. C. Excitonic switches operating at around 100 K. Nature Photon 2009, 3, $577-580$.

(38) Schmitz, A. C.; Ping, A. T.; Khan, M. A.; Chen, Q.; Yang, J. W.; Adesida, I. Schottky barrier properties of various metals on n-type GaN. Semicond. Sci. Technol. 1996, 11, $1464-1467$.

(39) Miura, N.; Nanjo, T.; Suita, M.; Oishi, T.; Abe, Y.; Ozeki, T.; Ishikawa, H.; Egawa, T.; Jimbo, T. Thermal annealing effects on Ni/Au based Schottky contacts on n-GaN and $\mathrm{AlGaN} / \mathrm{GaN}$ with insertion of high work function metal. Solid-State Electronics 2004, 48, 689-695.

(40) Rickert, K. A.; Ellis, A. B.; Kim, J. K.; Lee, J.-L.; Himpsel, F. J.; Dwikusuma, F.; Kuech, T. F. X-ray photoemission determination of the Schottky barrier height of metal contacts to n-GaN and p-GaN. J. Appl. Phys. 2002, 92, 6671-6678.

(41) Medjdoub, F.; Zegaoui, M.; Grimbert, B.; Rolland, N.; Rolland, P.-A. Effects of AlGaN Back Barrier on AlN/GaN-on-Silicon High-Electron-Mobility Transistors. Appl. Phys. Express 2011, 4, 124101.

(42) Zhang, X. B.; Taliercio, T.; Kolliakos, S.; Lefebvre, P. Influence of electron-phonon interaction on the optical properties of III nitride semiconductors. J. Phys.: Condens. Matter 2001, 13, 7053-7074.

(43) Schindler, C.; Zimmermann, R. Analysis of the exciton-exciton interaction in semiconductor quantum wells. Phys. Rev. B 2008, 78, 045313. 
(44) Laikhtman, B.; Rapaport, R. Correlations in a two-dimensional Bose gas with longrange interaction. Europhysics Letters 2009, 87, 27010.

(45) Rapaport, R.; Chen, G.; Simon, S. H. Nonlinear dynamics of a dense two-dimensional dipolar exciton gas. Phys. Rev. B 2006, 73, 033319.

(46) Stern, M.; Umansky, V.; Bar-Joseph, I. Exciton Liquid in Coupled Quantum Wells. Science 2014, 343, 55-57.

(47) Rosales, D.; Bretagnon, T.; Gil, B.; Kahouli, A.; Brault, J.; Damilano, B.; Massies, J.; Durnev, M. V.; Kavokin, A. V. Excitons in nitride heterostructures: From zero- to one-dimensional behavior. Phys. Rev. B 2013, 88, 125437.

(48) Andreani, L. C.; Tassone, F.; Bassani, F. Radiative lifetime of free excitons in quantum wells. Solid State Communications 1991, 77, 641-645.

(49) Butov, L. V.; Imamoglu, A.; Campman, K. L.; Gossard, A. C. Coulomb effects in spatially separated electron and hole layers in coupled quantum wells. J. Exp. Theor. Phys. 2001, 92, 260-266.

(50) Ivanov, A. L. Quantum diffusion of dipole-oriented indirect excitons in coupled quantum wells. EPL 2002, 59, 586-591.

(51) Vörös, Z.; Snoke, D. W.; Pfeiffer, L.; West, K. Direct Measurement of Exciton-Exciton Interaction Energy. Phys. Rev. Lett. 2009, 103, 016403-4.

(52) Remeika, M.; Graves, J. C.; Hammack, A. T.; Meyertholen, A. D.; Fogler, M. M.; Butov, L. V.; Hanson, M.; Gossard, A. C. Localization-Delocalization Transition of Indirect Excitons in Lateral Electrostatic Lattices. Phys. Rev. Lett. 2009, 102, 1868034.

(53) Remeika, M.; Leonard, J. R.; Dorow, C. J.; Fogler, M. M.; Butov, L. V.; Hanson, M.; 
Gossard, A. C. Measurement of exciton correlations using electrostatic lattices. Phys. Rev. B 2015, 92, 115311.

(54) Rossbach, G.; Levrat, J.; Jacopin, G.; Shahmohammadi, M.; Carlin, J. F.; Ganière, J. D.; Butté, R.; Deveaud, B.; Grandjean, N. High-temperature Mott transition in wide-band-gap semiconductor quantum wells. Phys. Rev. B 2014, 90, 201308. 